\title{
Addressing Spectral Narrowing in Cryogenic Yb:YAG: a 10 mJ Cryogenic Yb:YLF Regenerative Amplifier
}

\author{
M. Hemmer ${ }^{1}$, L. E. Zapata ${ }^{1}$, Y. Hua ${ }^{1}$, F. X. Kärtner ${ }^{1,2,3}$ \\ ${ }^{I}$ Center for Free-Electron Laser Science, Deutsches Elektronen Synchrotron,Notkestrasse 85, 22607 Hamburg, Germany \\ ${ }^{2}$ Department of Physics \& The Hamburg Center for Ultrafast Imaging, Universität Hamburg, Luruper Chaussee 149, \\ 22761 Hamburg, Germany \\ ${ }^{3}$ Research Laboratory of Electronics, Massachusetts Institute of Technology (MIT), Cambridge, Massachusetts 02139, USA \\ Author e-mail address: michael.hemmer@cfel.de
}

\begin{abstract}
We report a $\mathrm{Yb}: \mathrm{YLF}$ based $10 \mathrm{~mJ}$-class cryogenically-cooled regenerative amplifier with $2.1 \mathrm{~nm}$ FWHM spectral bandwidth. This system demonstrates the feasibility of high energy, sub-ps cryogenic lasers as an alternative to room temperature $\mathrm{Yb}$ :YAG.

OCIS codes: (140.3280) Laser amplifiers; (140.3538) Lasers, pulsed; (140.3480) Lasers, diode-pumped.
\end{abstract}

\section{Introduction}

The generation of high-energy pulses at high average power with sub-ps duration at 1- $\mu \mathrm{m}$ wavelength from diode pumped solid-state lasers is a critical area of laser development as it affects numerous applications. Indeed, lasers featuring such parameters find applications in fields as diverse as X-ray generation, material processing, nonlinear optical devices such as optical parametric chirped pulse amplifiers (OPCPAs) pumping and THz radiation generation and amplification. A number of architectures have been investigated toward the generation of simultaneous high average power and high energy, sub-ps duration optical pulses - mostly based on Yb:YAG gain medium - including room temperature thin-disk lasers [1] or room temperature crystal fiber [2]. Rod-type [3], slab [4] or composite thin-disk geometries [5] have also been investigated at cryogenic temperature and have resulted in the generation of $\mathrm{kW}$ average power at Joule level optical pulses [6]. The use of Yb:YAG at cryogenic temperature alleviates a number of shortcomings of the room temperature operation yet suffers from reduced emission bandwidth and therefore struggles to deliver sub-ps duration pulses.

Here, we follow up on previous work [7] to investigate the potential of cryogenic Yb:YLF as an alternative to Yb:YAG to deliver sub-ps duration pulses at high energy. We present a regenerative amplifier delivering up to 11 $\mathrm{mJ}$ energy with a spectral bandwidth supporting sub-750 fs duration optical pulses seeded by a homebuilt fiber frontend.

\section{Experimental layout}

The regenerative amplifier consists of a 4-mirror ring cavity arranged in bowtie geometry (Fig. 1). The seed pulses are provided by a home-built fiber laser frontend that delivers up to $\sim 100 \mathrm{~nJ}$ energy pulses at $38 \mathrm{MHz}$ repetition rate. The spectro-temporal properties of this frontend system were tailored to ideally seed a high energy amplifier chain: the pulses are temporally stretched to $1 \mathrm{~ns}$ duration and carry up to $2.4 \mathrm{~nm}$ of spectral bandwidth (FWHM) corresponding to a transform-limited duration of $\sim 500 \mathrm{fs}$ (for a sech ${ }^{2}$ temporal profile). The gain medium employed in the regenerative amplifier is a $1.75 \mathrm{~mm}$ thick, $25 \%$-doped Yb:YLF crystal with a $6 \times 8 \mathrm{~mm}^{2}$ clear aperture featuring two undoped caps. The crystallographic orientation of the gain medium is arranged to ensure amplification along the E//a axis. The crystal is mounted onto a copper heat sink attached to a liquid-nitrogen Dewar. The seed pulses are injected into and ejected from the regenerative amplifier cavity via a combination of a thin-film polarizer, a half-wave plate and a Pockels cell. The Pockels cell employed in our setup is operated in halfwave voltage and consists of a $1 \mathrm{~cm}$ diameter KD*P crystal. In this experiment, the high voltage switch of the Pockels cell limits the repetition rate of the amplifier system to $10 \mathrm{~Hz}$. The pump power is provided by a fiber coupled diode bar array emitting at $960 \mathrm{~nm}$ wavelength and providing up to $280 \mathrm{~W}$ continuous wave average power. The unpolarized output of the delivery fiber is split into two equal arms via a polarizing beam splitter and relay imaged to a flat-top $1.5 \mathrm{~mm}$ diameter spot via a three-lens imaging telescope onto the gain medium. The spectral output of the amplifier was recorded using a $0.1 \mathrm{~nm}$ spectral resolution spectrum analyzer and the spatial properties measured using a CCD camera. 


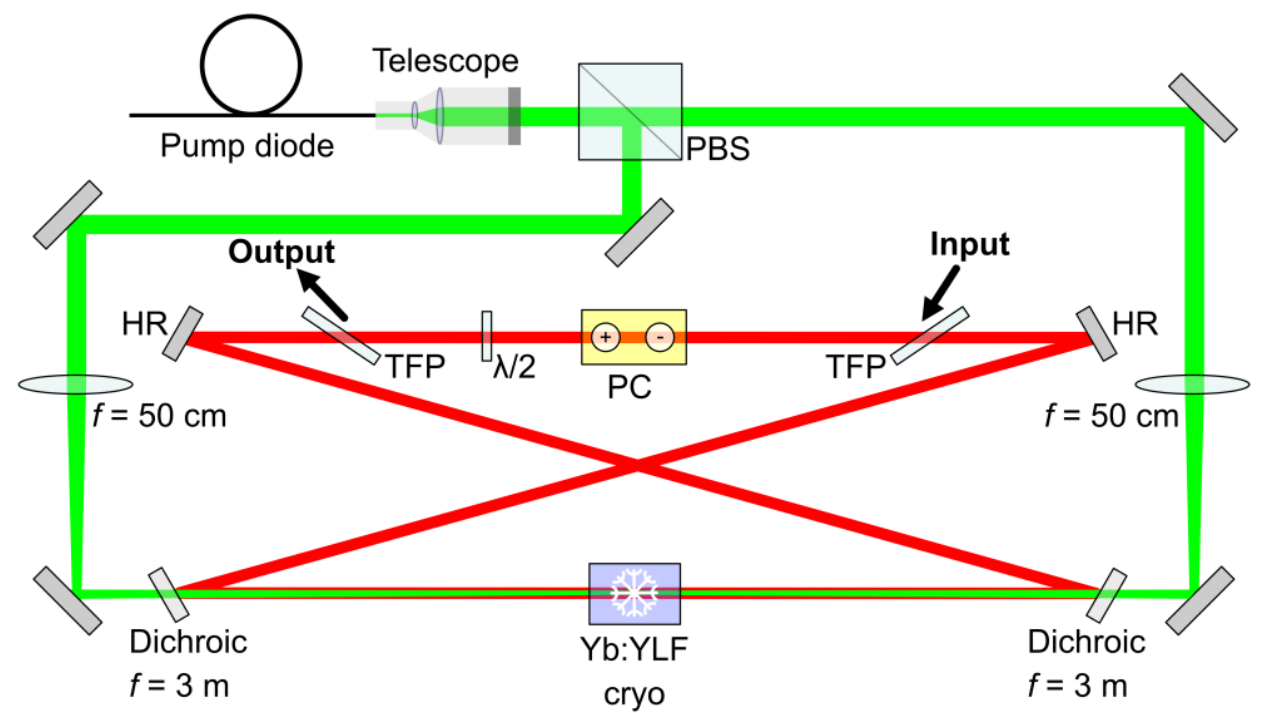

Fig. 1. Layout of the cryogenically-cooled Yb:YLF regenerative amplifier. HR: high reflectivity mirror, TFP: thin film polarizer, PC: Pockels cell, PBS: polarizing beam splitter.

\section{Laser performances}

The regenerative amplifier is operated in the unsaturated regime owing to the relatively low emission cross section of Yb:YLF $\left(\sigma_{\sigma}=1.2 \times 10^{-20} \mathrm{~cm}^{2}\right.$ at $\left.77 \mathrm{~K}[8]\right)$ - compared to $\sigma_{\mathrm{e}}=10 \times 10^{-20} \mathrm{~cm}^{2}$ for $\mathrm{Yb}: \mathrm{YAG}$ at $77 \mathrm{~K}$ - that place the saturation fluence above the damage fluence. An energy of $11 \mathrm{~mJ}$ is obtained at the output of the amplifier after 64 passes in the cavity for a seed energy of $3.5 \mathrm{~nJ}$. The diode pump source was set to operate gated at $280 \mathrm{~W}$ with a gate duration of $2 \mathrm{~ms}$. Increasing the seed energy to $21 \mathrm{~nJ}$ results in a reduction of the number of passes to yield the same $11 \mathrm{~mJ}$ output energy after 56 passes (Fig. 2 - left). We observe only limited spectral narrowing from $2.4 \mathrm{~nm}$ to $2.1 \mathrm{~nm}$ during the amplification process despite the net gain of $\sim 10^{6}$ (Fig. 2 - right), in good agreement with our numerical simulations. Notice that a comparable amplifier featuring a cryogenically cooled $\mathrm{Yb}$ :YAG gain medium showed a reduction of the seed spectrum down to $0.24 \mathrm{~nm}$ spectral width, supporting a $\sim 6.5$ ps duration (assuming a Gaussian temporal profile) [9]. The spatial profile of the amplified beam was recorded and shows a Gaussian distribution in the near field (Fig. 2 - left, inset).
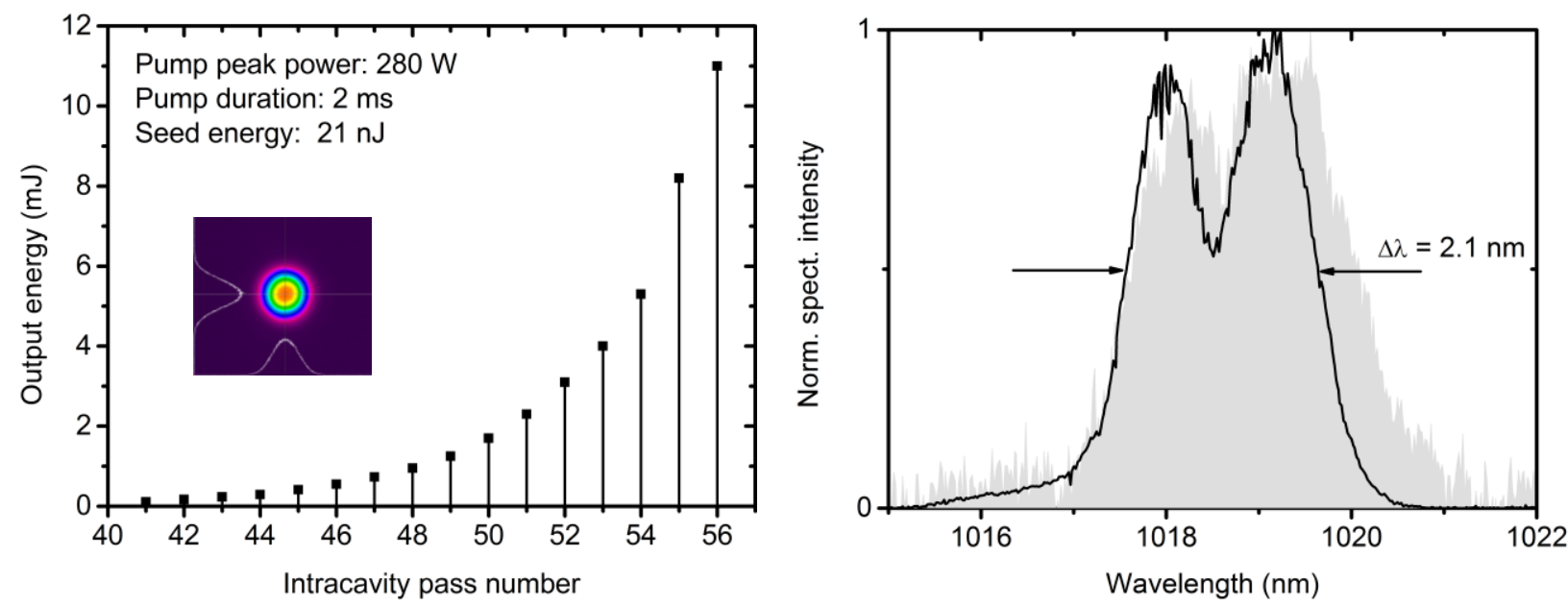

Fig. 2. (Left) Measured output energy versus number of passes in the regenerative amplifier cavity for a set pump peak power of $280 \mathrm{~W}$ and a set pump duration of $2 \mathrm{~ms}$ - inset: measured amplified output spatial profile in the near field; (right) measured output spectrum at $10 \mathrm{~mJ}$ energy (black line) and measured seed spectrum at the input of the regenerative amplifier (grey shadow).

\section{Conclusion \& future work}

We demonstrated a cryogenically cooled, diode pumped regenerative amplifier delivering up to $11 \mathrm{~mJ}$ energy and maintaining a spectral width supporting sub-ps duration with excellent spatial profile. Future work includes 
increasing the repetition rate to the $100 \mathrm{~Hz}$ regime, add a subsequent amplifier to boost the energy to the $100 \mathrm{~mJ}$ level and demonstrate temporal compression. This laser system is dedicated to drive nonlinear parametric devices.

\section{References}

[1] C. Teisset, M. Schultze, R. Bessing, M. Haefner, S. Prinz, D. Sutter, and T. Metzger, "300 W picosecond thin-disk regenrative amplifier at 10 kHz repetition rate," in Advanced Solid State Lasers (ASSL), 2013, p. paper JTh5A.1.

[2] X. Délen, Y. Zaouter, I. Martial, N. Aubry, J. Didierjean, C. Hönninger, E. Mottay, F. Balembois, and P. Georges, "Yb:YAG single crystal fiber power amplifier for femtosecond sources," Opt. Lett., vol. 38, no. 2, pp. 109-111, 2013.

[3] M. Hemmer, F. Reichert, K. Zapata, M. Smrz, A.-L. Calendron, H. Cankaya, K.-H. Hong, F. X. Kaertner, and L. E. Zapata, "Picosecond, 115 mJ energy, $200 \mathrm{~Hz}$ repetition rate cryogenic Yb:YAG bulk-amplifier," in Conference on Laser and Electro-Optics (CLEO), 2015, p. paper STu4O.3.

[4] S. Banerjee, K. Ertel, P. D. Mason, P. J. Phillips, M. De Vido, J. M. Smith, T. J. Butcher, C. Hernandez-Gomez, R. J. Greenhalgh, and J. L. Collier, "DiPOLE: a $10 \mathrm{~J}, 10 \mathrm{~Hz}$ cryogenic gas cooled multi-slab nanosecond Yb:YAG laser.," Opt. Express, vol. 23, no. 15, pp. 19542-51, 2015.

[5] L. E. Zapata, H. Lin, A.-L. Calendron, H. Cankaya, M. Hemmer, F. Reichert, W. R. Huang, E. Granados, K.-H. Hong, and F. X. Kärtner, "Cryogenic Yb:YAG composite-thin-disk for high energy and average power amplifiers," Opt. Lett., vol. 40, no. 11, pp. 2610-2613, 2015.

[6] C. M. Baumgarten, B. A. Reagan, M. Pedicone, H. Bravo, L. Yin, H. Wang, M. Woolston, B. Carr, C. Menoni, and J. Rocca, "Demonstration of a Compact $500 \mathrm{~Hz}$ Repetition Rate Joule-Level Chirped Pulse Amplification Laser," in Conference on Laser and Electro-Optics, 2016, p. paper STu3M.3.

[7] D. E. Miller, L. E. Zapata, D. J. Ripin, and Tso Yee Fan " Sub-picosecond pulses at $100 \mathrm{~W}$ average power from a Yb:YLF chirped-pulse amplification system," Optics Lett., vol. 37, no. 13, pp.2700-2702, 2012.

[8] T. Y. Fan, D. J. Rippin, R. L. Aggarwal, J. R. Ochoa, B. Chann, M. Tilleman, and J. Spitzberg, "Cryogenic Yb3+- doped Solid State Lasers," IEEE J. Sel. Top. Quantum Electron., vol. 13, no. 3, 2007.

[9] K. Hong, J. Gopinath, D. Rand, A. M. Siddiqui, S. Huang, E. Li, B. J. Eggleton, J. D. Hybl, T. Y. Fan, and F. X. Kärtner, "High-energy, kHzrepetition-rate, ps cryogenic Yb:YAG chirped pulse amplifier," Opt. Lett., vol. 35, no. 11, pp. 1752-1754, 2010. 